\title{
BMJ Open Clinical characteristics of young adult cataract patients: a 10-year retrospective study of the Zhongshan Ophthalmic Center
}

Duoru Lin, ${ }^{1}$ Zhenzhen Liu, ${ }^{1}$ Qianzhong Cao, ${ }^{1}$ Xiaohang Wu, ${ }^{1}$ Jinchao Liu, ${ }^{1}$ Jingjing Chen, ${ }^{1}$ Zhuoling Lin, ${ }^{1}$ Xiaoyan Li, ${ }^{1}$ Li Zhang, ${ }^{1}$ Erping Long, ${ }^{1}$ Xiayin Zhang, ${ }^{1}$ Jinghui Wang, ${ }^{1}$ Dongxuan Wu, ${ }^{2}$ Xutu Zhao, ${ }^{2}$ Tongyong Yu, ${ }^{2}$ Jing Li, ${ }^{1}$ Xiaojia Zhou, ${ }^{1}$ Lisha Wang, ${ }^{1}$ Haotian Lin, ${ }^{1}$ Weirong Chen, ${ }^{1}$ Yizhi Liu ${ }^{1}$

To cite: Lin D, Liu Z, Cao Q, et al. Clinical characteristics of young adult cataract patients: a 10-year retrospective study of the Zhongshan Ophthalmic Center. BMJ Open 2018;8:e020234. doi:10.1136/ bmjopen-2017-020234

$\mathrm{DL}$ and ZL contributed equally.

Received 23 0ctober 2017

Revised 2 May 2018

Accepted 22 June 2018
Check for updates

(C) Author(s) (or their employer(s)) 2018. Re-use permitted under CC BY-NC. No commercial re-use. See rights and permissions. Published by BMJ.

${ }^{1}$ State Key Laboratory of Ophthalmology, Zhongshan Ophthalmic Center, Sun Yat-sen University, Guangzhou, China

${ }^{2}$ Zhongshan School of Medicine, Sun Yat-sen University, Guangzhou, China

Correspondence to

Prof. Haotian Lin;

haot.lin@hotmail.com

\section{ABSTRACT}

Aim To investigate the characteristics of young adult cataract (YAC) patients over a 10-year period.

Methods This observational study included YAC patients aged 18-49 years who were treated surgically for the first time at the Zhongshan Ophthalmic Center in China. YAC patients were analysed and compared with patients with childhood cataract (CC) in January 2005 to December 2014.

Results During the 10-year period, 515 YAC patients and 2421 inpatients with CC were enrolled. Among the YAC patients, 76.76\% (109/142) of unilateral patients had a corrected distance visual acuity (CDVA) better than $20 / 40$ in the healthy eye, whereas only $20.38 \%(76 / 373)$ of bilateral patients had a CDVA better than 20/40 in the eye with better visual acuity. Compared with the CC group, the YAC group had a higher proportion of rural patients ( $40.40 \%$ vs $31.60 \%, p=0.001)$. Furthermore, the prevalence of other ocular abnormalities in YAC patients was higher than that in patients with CC $(29.71 \%$ vs $17.47 \%, \mathrm{p}<0.001$ ).

Conclusions A large proportion coming from rural areas and a high prevalence of complicated ocular abnormalities may be the most salient characteristics of YAC patients. Strengthening the counselling and screening strategy for cataract and health education for young adults are required especially for those in rural areas.

\section{INTRODUCTION}

Cataract remains the leading cause of blindness among visually impaired people in middle-income and low-income countries. ${ }^{1}$ Cataract affects people of any age, from childhood cataract (CC) ${ }^{2}$ to age-related cataract (ARC), ${ }^{3}$ which represent the leading causes of vision disabilities and treatable blindness in children and the general ageing population, respectively. Young adult cataract (YAC) is a special cataract population aged between 18-49 years old. ${ }^{4}$ Working-age young adults have always been regarded as

\section{Strengths and limitations of this study}

- This 10-year study may be the first to focus on the characteristics of young adult cataract (YAC) population and compared with patients with childhood cataract (CC).

- Limited by the study's research method, although we have made the best efforts to exclude age-related cataract (ARC) and CC from the YAC population, YAC may still have aetiological overlap with ARC and CC.

- Education level of parents and family income of YAC patients were unavailable in this retrospective study and were not analysed, and will be included in our following study series.

the breadwinners of their families, and their working ability, identity, self-respect and living conditions may be severely impacted by impaired visual function caused by YAC. ${ }^{5} 6$ However, YAC patients may be neglected and are rarely reported, potentially due to the relatively low disease prevalence, weak health awareness among the low-income population $^{7}$ and possibly certain unknown characteristics of this special population. The data on the characteristics of YAC patients are most likely to have clinical significance for health promotion and educational programmes and provide treatment strategies for cataract in this population. However, nearly all published reports have focused either on patients with $\mathrm{CC}$ or on patients with ARC. ${ }^{8-10}$ In the current study, we investigated the characteristics of YAC inpatients who were presented to the hospital in Guangzhou, China, between 18 and 49 years old, and compared these features with those of patients with CC during the same period. This study was examined over a 10 -year period and focused 
on the characteristics of YAC patients and could provide a useful reference for clinical management strategies of this neglected cataract population.

\section{METHODS \\ Patients}

This 10-year period observational study included inpatients with cataract aged between 18 and 49 years at the Zhongshan Ophthalmic Center (ZOC) in Guangzhou, China, from January 2005 to December 2014. Data on YAC hospitalisations were retrieved from the database of the Medical Records Department of the ZOC based on the three following specified codes of the International Classification of Diseases, 9th Revision, Clinical Modification: infantile cataract (366.0), aphakia and other disorders of the lens (379.3), and congenital cataract and lens anomalies (743.3). The inclusion criteria were set as follows: (1) patients with cataract aged 18-49 years old (the age range of YAC patients was previously defined as $18-49$ years $^{4}$; this is because the main purpose of this study was to investigate the characteristics of YAC patients, where age was the major inclusion criterion); (2) surgically treated for the first time; and (3) complete relevant patient data. Patients diagnosed with CC, ARC, traumatic cataract, complicated cataract, metabolic cataract and secondary cataract were excluded from the YAC population. Those with family history of cataract (diagnosed between 18 and 49 years old) were also excluded. The inclusion and exclusion criteria of each patient were judged according to the diagnosis, medical history, progress notes and other detailed medical records.

\section{Patient and public involvement}

No patients or the public were involved in the study protocol design, the specific aims or the research questions, and the plans for the design or implementation of the current study. No patients or the public were involved in the interpretation of the results of the study or preparation of the manuscript. There are no plans to disseminate the results of the research to study participants.

\section{Information extraction}

The eligible data of all patients were carefully reviewed by two independent researchers (DL and HL). The records and information of all patients were anonymised and de-identified prior to analysis. The demographics of YAC patients, including patients' numbers, gender, age, residence and length of stay, were extracted. Information on patients' eye conditions, including laterality, corrected distance visual acuity (CDVA), axial length (AL), surgical procedures and other isolated ocular anomalies, was also extracted and analysed. Amblyopia, a frequently complicated ocular anomaly in patients with $\mathrm{CC}^{11}$ was not included in the analysis of ocular anomalies due to the difficulties in the diagnosis prior to cataract surgery.

We compared the demographics and ocular situations of YAC patients with those of patients with CC within the same period to deliver a better presentation. ${ }^{12}$ Both populations were patients with cataract with impaired visual function from different ages (from childhood to young adult) and may have some associations. The similar clinical presentation (poor visual acuity, similar cataract morphology under slit lamp examination and abnormal ocular developments), as well as the same medical institution of treatment and study period, made the two populations comparable. The characteristics of the demographics and ocular situations of YAC patients would be clearer after comparing with those of patients with CC. The demographics and ocular situations of patients with CC of the same period have been reported in previous studies ${ }^{12}$ and these data were rearranged and reanalysed according to the same criteria used in the current study for additional scientific comparisons.

\section{Statistical analysis}

The data of all analysed patients with cataract were input into a purpose-built database in Microsoft Excel (Microsoft, Redmond, Washington, USA) spreadsheets, sorted and analysed by another two coresearchers (JC and ZLin), and mutually checked. The data were analysed using the Statistical Package for the Social Sciences (SPSS V.19.0). The age, CDVA and AL are presented as mean \pm SD. Gender, residence, laterality, surgical procedures and other isolated ocular anomalies are presented as absolute frequency (n) and relative frequency (\%). The ALs of the cataractous eyes and those of the contralateral healthy eyes in unilateral YAC patients were compared using paired t-test. The comparison of the rates of rural patients, native patients (from Guangdong Province), unilateral involvements and other ocular abnormalities between patients with CC and YAC patients were analysed using Pearson's $X^{2}$ test. A $p$ value $<0.05$ was considered statistically significant for all tests.

\section{RESULTS}

\section{The status of YAC inpatients}

During the 10-year period, 515 YAC inpatients were included. The mean age was $28.88 \pm 8.83$ years, and the male to female ratio was 1.2:1 (279:236). The distribution of the number and proportion (number of YAC patients/total number of patients with cataract) of YAC inpatients during the period of 10 years between 2005 and 2014 is shown in figure 1. Most of the patients came from South China and Central China (figure 2). Based on the eligible patients, $27.57 \%(142 / 515)$ were presented with unilateral cataract, $14.76 \%(76 / 515)$ in the left eye and $12.82 \%(66 / 515)$ in the right eye. Cataract extraction with intraocular lens (IOL) implantation was performed in 502 patients $(502 / 515,97.48 \%)$. However, the remaining 13 patients $(2.52 \%)$ underwent cataract extraction without IOL implantation due to bad preoperative CDVA (from no light perception (NLP) to hand movement $(\mathrm{HM}) / 10 \mathrm{~cm})$. There were two purposes for performing cataract extraction in these 13 patients: (1) 
A

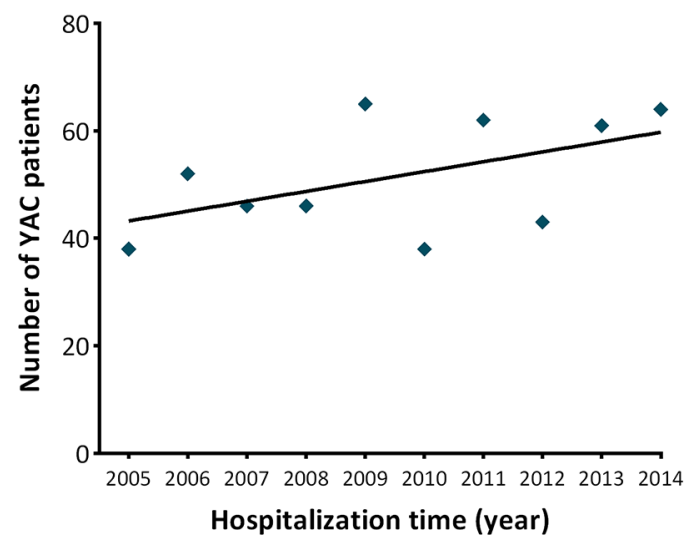

B

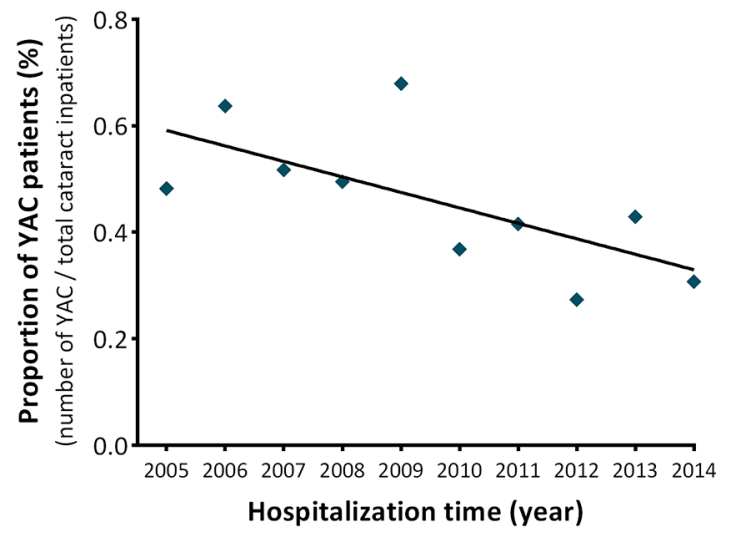

Figure 1 Distribution of the number and proportion of YAC inpatients from 2005 to 2014 . The fitted lines show an uptrend (A) and downtrend (B). YAC, young adult cataract.

removing the cloudy lens so as to perform further fundus examination and treatment; and (2) eliminating the 'white-pupil' to improve the ocular appearance of these young adults.

In the visual acuity analysis, nearly $80 \%$ of the unilateral patients $(76.76 \%, 109 / 142)$ had one eye with a CDVA better than 20/40. Among the bilateral patients (with analysis performed in the eye with a better visual acuity), only $20.38 \%(76 / 373)$ had a CDVA better than 20/40. Furthermore, nearly $10 \%(9.92 \%, 37 / 373)$ of the bilateral patients had a CDVA worse than finger count (FC). $\mathrm{AL}$ is another important index for eye development, and the distribution of AL is shown in figure 3. Although no difference in AL between two eyes was revealed in bilateral patients $(24.82 \pm 2.75$ vs $24.70 \pm 2.65, \mathrm{t}=1.90, \mathrm{p}=0.058)$, the ALs of the cataractous eyes in unilateral patients were larger than those of the contralateral healthy eyes (25.23 \pm 2.43 vs $23.98 \pm 1.43, \mathrm{t}=5.05, \mathrm{p}<0.001)$.

Other complicated ocular abnormalities were significantly related to the prognosis of patients with cataract. According to case records, $29.71 \%(153 / 515)$ of YAC patients were confirmed to have at least one other ocular abnormality. The most common ocular anomaly was strabismus (exotropia and esotropia), which was recorded in 47 patients, with a prevalence of $9.13 \%$ (47/515). The second most common ocular abnormality in YAC patients was nystagmus $(8.16 \%, 42 / 515)$. The prevalence of anomalies of the iris (iridocoloboma and aniridia) was
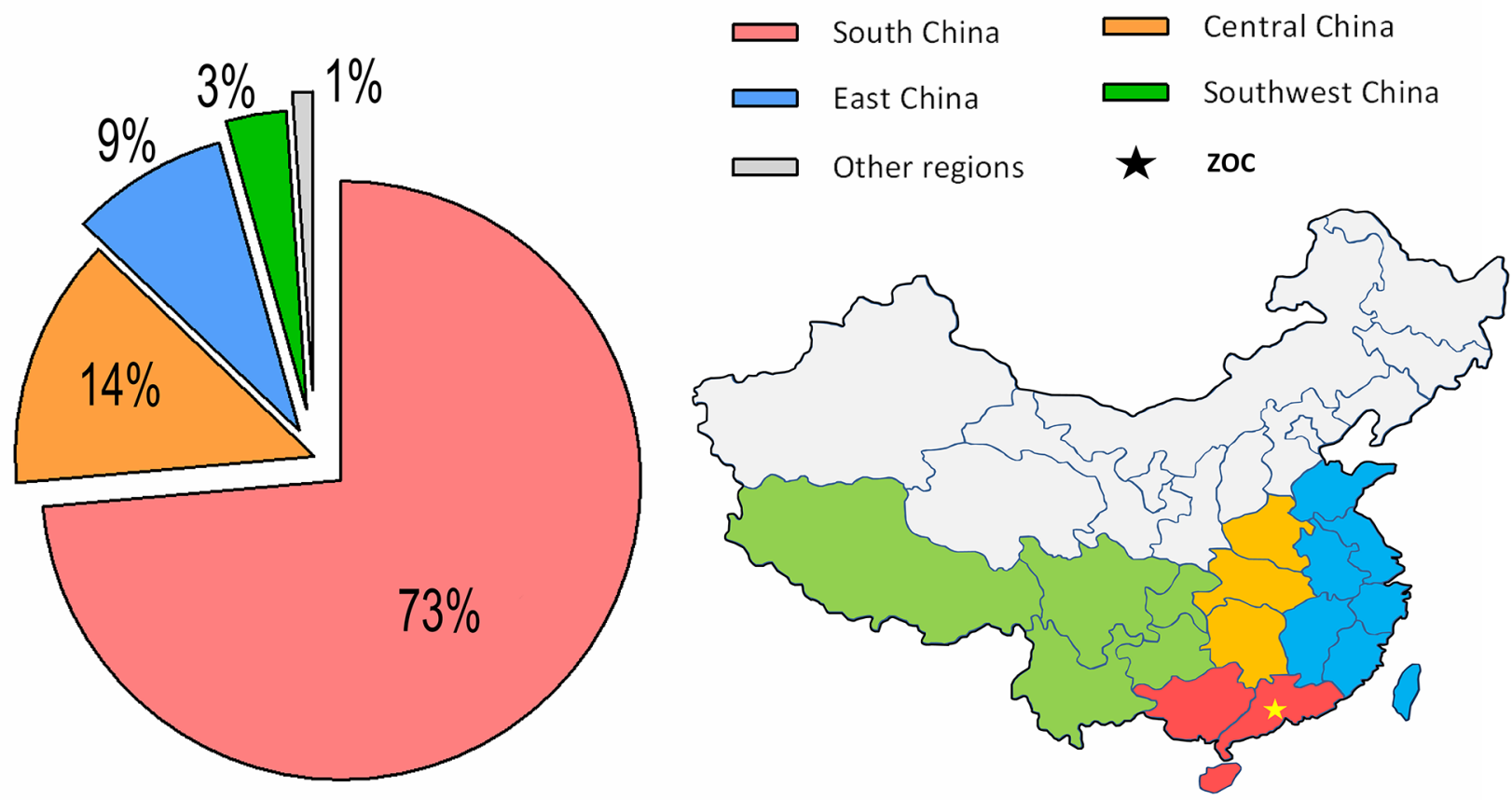

Figure 2 Residences of YAC inpatients. Most patients came from South China and Central China. YAC, young adult cataract; ZOC, Zhongshan Ophthalmic Center. 

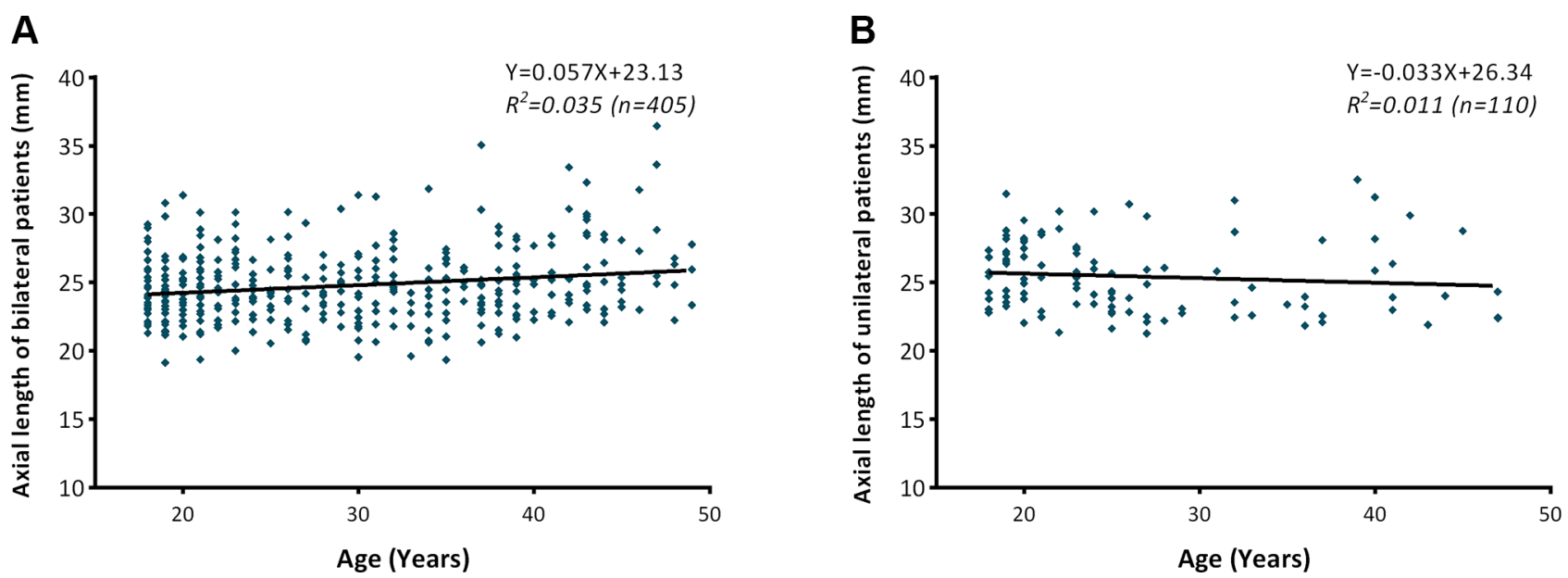

Figure 3 Distribution of the axial length of bilateral and unilateral YAC inpatients. The development of the axial length of YAC patients is in a relatively steady state. The right eye was arbitrarily selected to represent the ALs of bilateral individuals because no difference of $A L$ between the two eyes was found. YAC, young adult cataract.

$2.52 \%(13 / 515)$, accounting for the third most frequent ocular anomaly. Retina diseases $(2.14 \%, 11 / 515)$, vitreous body diseases $(1.94 \%, 10 / 515)$ and high myopia $(1.75 \%$, $9 / 515)$ were the following three most common ocular anomalies according to the medical records in this study.

\section{Comparisons of YAC patients and patients with CC}

To better analyse the characteristics of YAC patients, we compared the demographics and ocular situations with the data of patients with CC from previous studies, ${ }^{12}$ which were rearranged and reanalysed according to the same criteria used in the current study. As shown in table 1, the male to female ratio in YAC patients was lower than that in patients with CC. However, a larger proportion of patients from rural areas $(>40 \%)$, a smaller proportion of unilateral cataract and a larger proportion of patients undergoing cataract extraction with IOL implantation were revealed in YAC patients compared with patients with CC. Furthermore, the prevalence of other ocular abnormalities in YAC patients was higher than that in patients with CC (29.71\% vs $17.47 \%)$. Strabismus and nystagmus were the two most common ocular abnormalities in both groups. Other complicated ocular abnormalities in the two age groups are listed in figure 4 .

\section{DISCUSSION}

Compared with patients with $\mathrm{CC}$ and $\mathrm{ARC}$, who receive a great deal of disease management attention, YAC patients are an understudied population. The purpose of this study was to explore the characteristics of YAC patients, which is important for planning appropriate management strategies for this age group of patients with cataract.

An uptrend in patient numbers and downtrend in the proportion of YAC patients were revealed during the recent 10-year period, and more than $30 \%$ of YAC inpatients were from the regions of China other than their resident districts (Guangdong Province). Similar findings were obtained for patients with CC assessed in our prior study. These trends are due to increased national awareness and screening for cataracts, which resulted from the establishment of the Childhood Cataract Program of the

Table 1 Comparison of the characteristics of the two groups

\begin{tabular}{lllrl} 
& Patients with CC & YAC patients & Pearson $\chi^{2}$ & P values \\
\hline Number of inpatients with CC & 2421 & 515 & - & - \\
Male:female & $1.56: 1$ & $1.18: 1$ & 8.25 & $0.005^{*}$ \\
Guangdong Province (\%) & 69.43 & 67.77 & 0.55 & 0.460 \\
Rural area (\%) & 31.60 & 40.40 & 13.37 & $0.001^{*}$ \\
Unilateral CC inpatients (\%) & 33.75 & 27.57 & 7.36 & $0.007^{\star}$ \\
Age at surgery (years) & $3.73 \pm 4.68$ & $28.88 \pm 8.83$ & - & $<0.001 \dagger$ \\
Cataract extraction with IOL implantation (\%) & 65.51 & 97.48 & 211.25 & $<0.001^{*}$ \\
Other ocular abnormalities (\%) & 17.47 & 29.71 & 40.32 & $<0.001^{*}$ \\
\hline
\end{tabular}

*Pearson's $\chi^{2}$ test, with $p<0.05$ regarded as statistically significant. 


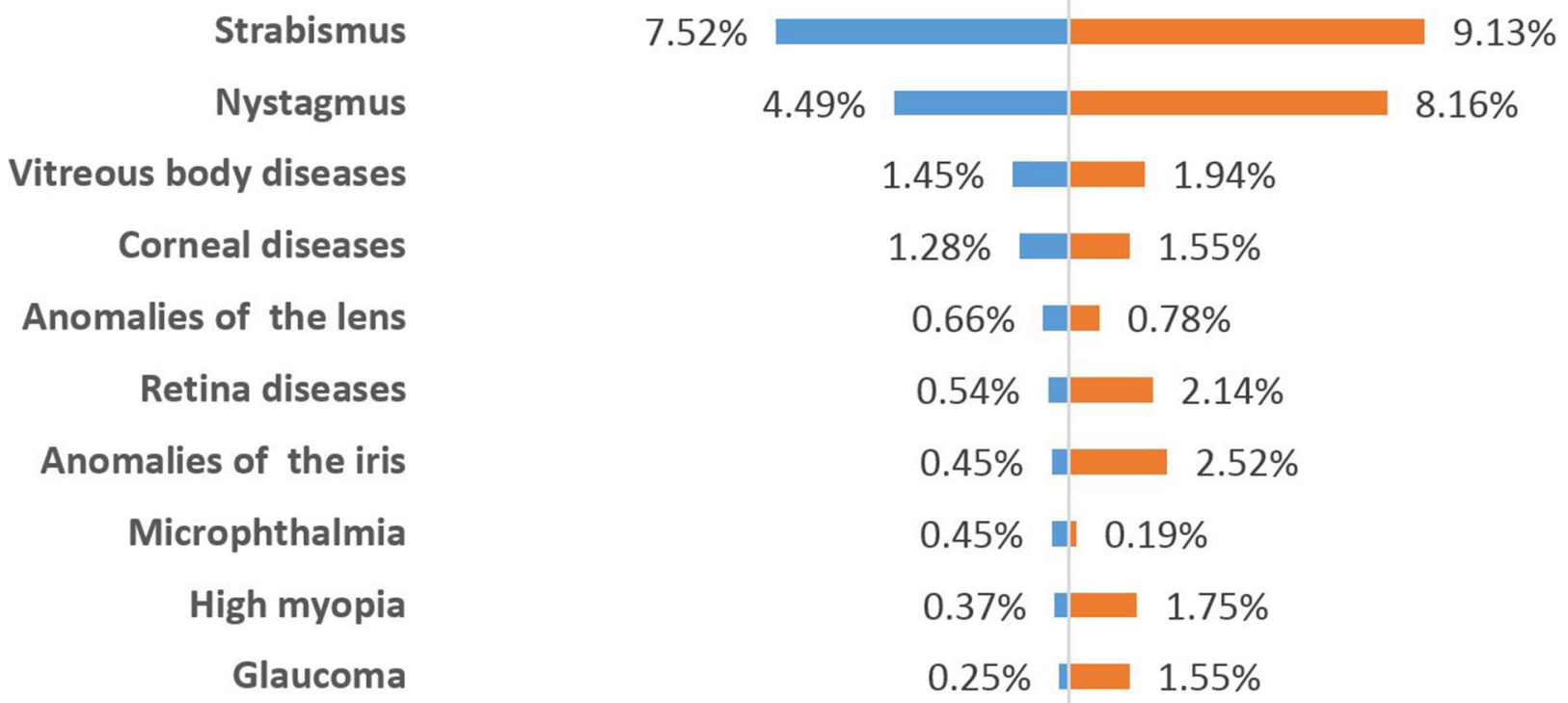

Figure 4 Comparison of other ocular abnormalities in YAC and CC inpatients. Strabismus and nystagmus were the two most frequent ocular abnormalities in YAC patients. CC, childhood cataract; YAC, young adult cataract.

Chinese Ministry of Health (CCPMOH) ${ }^{13}$ The downward trend in the proportion of YAC inpatients also results from the increasing rate of cataract surgery in China recently. ${ }^{14}$ However, the increase in the number of cataract cases may also result from rubella ${ }^{15}$ infection and low birth weight. ${ }^{1617}$ Despite the similarities in the residences and increasing number of patients, the proportion of male among YAC inpatients treated surgically for the first time was lower than that among inpatients with CC. This significantly lower proportion of male in YAC inpatients may be attributed to the traditional preference for sons in China; boys diagnosed with cataract were preferentially sent to hospitals for further treatment at a very young age. In contrast to patients with CC, more than $97 \%$ of YAC patients underwent primary IOL implantation. IOL implantation is the standard of care for both adult and paediatric cataract surgery, and the steady state of AL distribution shown in this study may indicate a relatively small postoperative refractive change.

The ZOC is a specialist eye care facility located in the first-tier city of Guangzhou; as such, the proportion of patient should be primarily urban. However, it is noteworthy that more than $40 \%$ of YAC patients came from rural areas, which was significantly higher than that of patients with CC. The low parental education level and family income of patients with CC from rural areas were shown in our previous study. ${ }^{18}$ Low health awareness and poor economic conditions might be the main barriers preventing YAC patients from the timely detection and treatment of the disease. Unfortunately, information on education and family income was not available in this retrospective study. Furthermore, the use of traditional biomass cooking fuels in rural areas may be another factor that contributes to cataract formation in YAC patients, although the association could have been the result of uncontrolled founding. ${ }^{4}$ Regardless of the reason, the large proportion of rural YAC patients observed in this study indicates that providing intensive counselling and improving the understanding of cataracts through country-based health education programmes may be beneficial for the timely detection and treatment of YAC patients.

A relatively good CDVA in unilateral patients or poor CDVA in bilateral patients was one of the salient characteristics of YAC patients. For unilateral patients, cataracts were frequently not realised or easily found due to the relatively good visual acuity in the contralateral eyes. Nearly $80 \%$ of unilateral patients in this study had a CDVA better than 20/40 in the healthy eye. These patients may believe that a relatively good visual acuity in one eye is sufficient for daily life and simple work, thereby resulting in delayed presentation to hospitals. These patients urgently require ophthalmological education because good vision encompasses far more than relatively good monocular visual acuity. ${ }^{1920}$ In addition, poor visual acuity may prevent bilateral patients from seeking timely treatment. In this study, only $20.38 \%$ (76/373) of bilateral patients had a CDVA better than 20/40 in the eye with better visual acuity, and nearly $10 \%$ of these patients had a CDVA worse than FC. These patients may be accustomed to low vision that is left untreated for a long time, which ultimately may lead them to seek surgical treatment due to severely worsened visual acuity. For young adults with any abnormal visual acuity, detailed ophthalmic examinations are required to rule out YAC.

Complicated eye conditions represent another dominant trait of YAC patients. The prevalence of ocular anomalies among all YAC patients was $29.71 \%$, which was approximately twice the rate of patients with CC. ${ }^{12}$ 
Strabismus and nystagmus were the two most common ocular abnormalities in both YAC patients and patients with CC. Although we had made our best efforts to exclude CC from the YAC population, patients with other comorbid conditions were likely to be developmental and need to be further explored in this study. Complicated ocular abnormalities frequently indicate poor visual prognosis $^{21}{ }^{22}$; thus, a detailed explanation of potential poor postoperative visual function is needed for YAC patients who are presented with related ocular complications. More importantly, timely treatment remains one of the most effective measures to improve postoperative visual function in YAC patients with ocular abnormalities.

\section{Limitations}

Several factors could limit the extent to which this study's results can be generalised. First, there was not much existing literature to rely on or refer to because the YAC population has rarely been studied. Thus, we compared the characteristics of YAC with those of patients with CC to make the characteristics of demographics and ocular situations of YAC patients more apparent. Second, limited by the research method of this study, although we have used best efforts to exclude ARC and CC from the YAC population based on the age of onset, morphology of lens opacity and detailed medical records, there may still have aetiological overlap between YAC and ARC and CC. Third, education and family income levels of YAC patients were not analysed due to the unavailability of these data in this retrospective study. These factors will be included in our following prospective study series for further analysis.

\section{CONCLUSIONS}

Earlier diagnosis and treatment are extremely vital to restore vision early and to reduce the financial burden that follows among those with long-standing cataracts and dense amblyopia. Target prevention and treatment strategies based on the findings of this study may be beneficial for the timely detection and treatment of YAC patients, which includes providing intensive counselling and improving the understanding of cataract through country-based health education programmes, and by providing detailed ophthalmic examinations for young adults with any abnormal visual acuity to rule out YAC. This article represents only the beginning of YAC patient studies, and the management strategy of this population still requires further investigation, as well as more funding and medical resource support.

Acknowledgements We thank Deying Yu for improving the quality of English of the manuscript.

Contributors HL, DL, WC and YL contributed to the conception or design of the work. ZLiu, QC, XW, JLiu, JC, ZLin and XL contributed to the acquisition of data. LZ, EL, XZha and JW contributed to the analysis. DW, XZ, TY, JLi, XZhao and LW contributed to the interpretation of data for the work. HL and DL contributed to drafting the work and critical revision for important intellectual content. All authors gave final approval of the version to be published.
Funding This clinical study was supported by the NSFC $(81270980,81300750)$, the Guangdong Provincial Natural Science Foundation for Distinguished Young Scholars of China (2014A030306030), and the Clinical Research and Translational Medical Center of Pediatric Cataract in Guangzhou City (201505032017516). The sponsors of the study played no role in the study protocol design, data collection, data analysis, data interpretation, manuscript preparation or the decision to submit the manuscript for publication.

Competing interests None declared.

Patient consent Not required.

Ethics approval All procedures in our series of ongoing cataract studies were consistent with the tenets of the Declaration of Helsinki and were approved by the Ethical Review Committee of the Zhongshan Ophthalmic Center in Sun Yat-sen University (ERC-ZOC-SYSU). Due to the retrospective nature of the study, written informed consent was waived by the ERC.

Provenance and peer review Not commissioned; externally peer reviewed.

Data sharing statement All of the data are in the manuscript, and no additional data are available.

Open access This is an open access article distributed in accordance with the Creative Commons Attribution Non Commercial (CC BY-NC 4.0) license, which permits others to distribute, remix, adapt, build upon this work non-commercially, and license their derivative works on different terms, provided the original work is properly cited, appropriate credit is given, any changes made indicated, and the use is non-commercial. See: http://creativecommons.org/licenses/by-nc/4.0/.

\section{REFERENCES}

1. Liu YC, Wilkins M, Kim T, et al. Cataracts. Lancet 2017;390:600-12.

2. Sheeladevi S, Lawrenson JG, Fielder AR, et al. Global prevalence of childhood cataract: a systematic review. Eye 2016;30:1160-9.

3. Fukuoka $\mathrm{H}$, Afshari NA. The impact of age-related cataract on measures of frailty in an aging global population. Curr Opin Ophthalmol 2017;28:93-7.

4. Tanchangya J, Geater AF. Use of traditional cooking fuels and the risk of young adult cataract in rural Bangladesh: a hospital-based case-control study. BMC Ophthalmol 2011;11:16.

5. Cillino G, Casuccio A, Pasti M, et al. Working-age cataract patients: visual results, reading performance, and quality of life with three diffractive multifocal intraocular lenses. Ophthalmology 2014;121:34-44.

6. Li YJ, Xirasagar S, Pumkam C, et al. Vision insurance, eye care visits, and vision impairment among working-age adults in the United States. JAMA Ophthalmol 2013;131:499-506.

7. Lin $\mathrm{H}$, Lin $\mathrm{D}$, Long $\mathrm{E}$, et al. Patient participation in free cataract surgery: a cross-sectional study of the low-income elderly in urban China. BMJ Open 2016;6:e011061.

8. Lambert SR, Lynn MJ, DuBois LG, et al. Axial elongation following cataract surgery during the first year of life in the infant Aphakia Treatment Study. Invest Ophthalmol Vis Sci 2012;53:7539-45.

9. Lin H, Lin D, Liu Z, et al. A Novel Congenital Cataract Category System Based on Lens Opacity Locations and Relevant Anterior Segment Characteristics. Invest Ophthalmol Vis Sci 2016;57:6389-95.

10. Lin H, Lin D, Chen J, et al. Distribution of Axial Length before Cataract Surgery in Chinese Pediatric Patients. Sci Rep 2016;6:23862.

11. Rajavi Z, Mokhtari S, Sabbaghi H, et al. Long-term visual outcome of congenital cataract at a Tertiary Referral Center from 2004 to 2014. J Curr Ophthalmol 2015;27:103-9.

12. Lin D, Chen J, Lin Z, et al. 10-Year Overview of the Hospital-Based Prevalence and Treatment of Congenital Cataracts: The CCPMOH Experience. PLoS One 2015;10:e0142298.

13. Lin $\mathrm{H}$, Chen $\mathrm{W}$, Luo L, et al. Ocular hypertension after pediatric cataract surgery: baseline characteristics and first-year report. PLoS One 2013;8:e69867.

14. Zhu M, Zhu J, Lu L, et al. Four-year analysis of cataract surgery rates in Shanghai, China: a retrospective cross-sectional study. BMC Ophthalmol 2014;14:3.

15. Singh MP, Ram J, Kumar A, et al. Infectious agents in congenital cataract in a tertiary care referral center in North India. Diagn Microbiol Infect Dis 2016;85:477-81.

16. SanGiovanni JP, Chew EY, Reed GF, et al. Infantile cataract in the collaborative perinatal project: prevalence and risk factors. Arch Ophthalmol 2002;120:1559-65. 
17. Prakalapakorn SG, Rasmussen SA, Lambert SR, et al. Assessment of risk factors for infantile cataracts using a case-control study: National Birth Defects Prevention Study, 2000-2004. Ophthalmology 2010;117:1500-5.

18. Chen J, Lin D, Lin Z, et al. Height, weight and body mass index of children with congenital cataracts before surgical treatment. $B M C$ Ophthalmol 2017:17:119.

19. Brian G, Taylor H. Cataract blindness--challenges for the 21st century. Bull World Health Organ 2001;79:249-56.
20. Parker AJ. Vision in our three-dimensional world. Philosophical transactions of the Royal Society of London Series B, Biological sciences, 2016:371.

21. Trumler AA. Evaluation of pediatric cataracts and systemic disorders. Curr Opin Ophthalmol 2011;22:365-79.

22. Sisk RA, Berrocal AM, Feuer WJ, et al. Visual and anatomic outcomes with or without surgery in persistent fetal vasculature. Ophthalmology 2010;117:2178-83. 